\title{
Coding of Stereoscopic Depth Information in Visual Areas V3 and V3A
}

\author{
Akiyuki Anzai, ${ }^{1}$ Syed A. Chowdhury, ${ }^{2}$ and Gregory C. DeAngelis ${ }^{1}$ \\ ${ }^{1}$ Department of Brain and Cognitive Sciences, Center for Visual Science, University of Rochester, Rochester, New York 14627, and ${ }^{2}$ Department of \\ Neurobiology, Washington University School of Medicine, St. Louis, Missouri 63110
}

The process of stereoscopic depth perception is thought to begin with the analysis of absolute binocular disparity, the difference in position of corresponding features in the left and right eye images with respect to the points of fixation. Our sensitivity to depth, however, is greater when depth judgments are based on relative disparity, the difference between two absolute disparities, compared to when they are based on absolute disparity. Therefore, the visual system is thought to compute relative disparities for fine depth discrimination. Functional magnetic resonance imaging studies in humans and monkeys have suggested that visual areas V3 and V3A may be specialized for stereoscopic depth processing based on relative disparities. In this study, we measured absolute and relative disparity-tuning of neurons in V3 and V3A of alert fixating monkeys, and we compared their basic tuning properties with those published previously for other visual areas. We found that neurons in $\mathrm{V} 3$ and $\mathrm{V} 3 \mathrm{~A}$ predominantly encode absolute, not relative, disparities. We also found that basic parameters of disparity-tuning in V3 and V3A are similar to those from other extrastriate visual areas. Finally, by comparing single-unit activity with multi-unit activity measured at the same recording site, we demonstrate that neurons with similar disparity selectivity are clustered in both V3 and V3A. We conclude that areas V3 and V3A are not particularly specialized for processing stereoscopic depth information compared to other early visual areas, at least with respect to the tuning properties that we have examined.

\section{Introduction}

Localizing objects in three-dimensional (3D) space and recognizing them according to 3D shape are important yet complex tasks that the visual system performs remarkably well. Many cues are available for these tasks, but binocular disparity is a particularly robust cue (Wheatstone, 1838; Julesz, 1971). Absolute binocular disparity refers to the difference in location between corresponding features in the left and right eye images relative to the points of visual fixation. Although absolute disparity is a sufficient cue for stereoscopic depth perception (Julesz, 1971), our sensitivity to small variations in depth is significantly enhanced when depth judgments are based on relative disparity, the difference between two absolute disparities (Westheimer, 1979; Erkelens and Collewijn, 1985). This suggests that the visual system computes relative disparity and uses it for fine-scale depth judgments (Westheimer, 1979).

Neural mechanisms for encoding binocular disparity in the primary visual cortex (V1) have been well studied (for review, see Cumming and DeAngelis, 2001), and many neurons in cortical

\footnotetext{
Received Nov. 11, 2010; revised May 23, 2011; accepted May 26, 2011.

Author contributions: G.C.D. designed research; A.A. and S.A.C. performed research; A.A. analyzed data; A.A. and G.C.D. wrote the paper.

This work was supported by a research grant (EY013644) and a Core grant (EY001319) from the National Eye Institute. We thank Dina-Jo Knoedl and Swati Shimpi for assistance with animal care and training and Tunde Adeyemo and Swati Shimpi for processing MRl images for area registration.

Correspondence should be addressed to Dr. Gregory C. DeAngelis, Center for Visual Science, University of Rochester, 315 Meliora Hall, Rochester, NY 14627. E-mail: gdeangelis@cvs.rochester.edu.

S. A. Chowdhury's present address: Biology Department, St. Louis Community College at Wildwood, Wildwood, M0 63040 .

DOI:10.1523/JNEUROSCI.5956-10.2011

Copyright $\odot 2011$ the authors $\quad 0270-6474 / 11 / 3110270-13 \$ 15.00 / 0$
}

areas along both dorsal and ventral processing streams exhibit selectivity for binocular disparity (for review, see Gonzalez and Perez, 1998; Parker, 2007) (see also Neri, 2005). In contrast, neural mechanisms for computing relative disparity are not as well understood. In V1 and the middle temporal (MT) area, neurons are tuned to absolute disparity (Cumming and Parker, 1999; Uka and DeAngelis, 2006). A small fraction of neurons in V2 have been reported to be selective for relative disparity, but most are selective for absolute disparity (Thomas et al., 2002). In area V4, many neurons show a greater degree of relative disparity selectivity, yet a majority of neurons are only partially selective for relative disparity (Umeda et al., 2007). This suggests that relative disparity processing may gradually progress along the ventral stream (cf. Neri, 2005; Parker, 2007). However, other areas need to be explored before definitive conclusions can be drawn. In particular, areas V3 and V3A are of considerable interest as they have been implicated by functional magnetic resonance imaging (fMRI) studies (Backus et al., 2001; Tsao et al., 2003) (but see Neri et al., 2004) as being specialized for relative disparity processing. However, basic quantitative accounts of disparity-tuning properties for V3 and V3A neurons in behaving monkeys are very limited (Poggio et al., 1988), and it is unknown whether V3 and V3A neurons show selectivity for relative disparity.

In light of these facts, we measured disparity-tuning of neurons in V3 and V3A of behaving monkeys, using center-surround random-dot stimuli to examine whether they show selectivity for relative disparity. We also quantified basic disparity-tuning characteristics of V3 and V3A neurons and compared these with data from other cortical areas reported previously. We then compared single-unit (SU) and multi-unit (MU) responses measured at the 
same recording sites to determine whether neurons are clustered according to their disparity-tuning characteristics. Our results, unlike the fMRI studies, provide no indication that areas V3 and V3A are specialized for processing stereoscopic depth information.

\section{Materials and Methods}

Experiments were performed on two young adult male rhesus monkeys (Macaca mulatta). All surgical and experimental procedures complied with guidelines for the use and care of laboratory animals set by the U.S. National Institutes of Health (Bethesda, MD) and were approved by the Animal Studies Committee at Washington University School of Medicine (St. Louis, MO) and by the University Committee on Animal Resources at the University of Rochester (Rochester, NY).

Surgical preparation. A series of aseptic surgeries were performed on each animal (for details, see Uka and DeAngelis, 2003) to prepare for behavioral training and physiological recording experiments. To immobilize the animal's head during experiments, a head post receptacle was affixed to the skull with dental acrylic. Eye coils were implanted for monitoring the positions of both eyes, thus allowing computation of both version and vergence angles. Based on brain images obtained from structural magnetic resonance imaging (sMRI), the locations of areas V3 and V3A were estimated by mapping a surface-based atlas onto the sMRI images using CARET (Computerized Anatomical Reconstruction and Editing Toolkit) software (Van Essen et al., 2001). Subsequently, a small craniotomy $(\sim 19 \mathrm{~mm}$ in diameter $)$ was made over the lunate sulcus $\sim 15$ $\mathrm{mm}$ lateral to the midline and $14 \mathrm{~mm}$ anterior to the occipital ridge to allow access to these areas with microelectrodes. A recording chamber was then mounted over the craniotomy in a parasagittal plane.

Behavioral tasks and visual stimuli. Each animal's daily water intake was controlled to motivate the animal to perform behavioral tasks for liquid reward. Each animal was trained to fixate on a small visual target $\left(0.2^{\circ}\right)$ presented on a display screen $(87.4 \times 70.5 \mathrm{~cm})$ placed $57 \mathrm{~cm}$ in front of the animal. Each trial began when a fixation marker appeared at the center of the visual display. If the animal fixated on the marker and kept gaze within a $1.5^{\circ} \times 1.5^{\circ}$ fixation window, a visual stimulus was then displayed for $1.5 \mathrm{~s}$. The animal received a liquid reward if he kept his gaze within the fixation window throughout the stimulus presentation; otherwise, the trial was aborted and the data discarded. Ocular vergence was relatively stable across trials. We computed the standard deviation of vergence angle across trials for each recording session, and the mean value of this standard deviation across all sessions was $0.22^{\circ}$ for both animals.

Visual stimuli were generated using the OpenGL graphics library in conjunction with an accelerated video graphics card (nVIDIA QuadroFX 1000 or 1400), and stimuli were rear-projected onto the display screen by a stereoscopic projector (Christie Digital Mirage 2000). Stereoscopic stimuli were presented by displaying left and right eye half-images alternately at a refresh rate of $100 \mathrm{~Hz}$, and the animal viewed the display through a pair of ferroelectric liquid crystal shutter glasses (Displaytech) that were synchronized with the video refresh.

Electrophysiological recordings. At the beginning of each recording session, a cylindrical recording grid (Crist Instruments) containing a regular array of holes (1 mm apart) was mounted onto the recording chamber and a stainless steel guide tube was inserted through one of the holes to penetrate the dura mater. An epoxy-coated tungsten microelectrode $(\sim 3$ $\mathrm{M} \Omega$; FHC Inc.) was inserted into the guide tube and advanced through the cortex using an oil hydraulic micromanipulator (Narishige) to record extracellular signals from single neurons. The neural signals were amplified, filtered, passed through a dual time-amplitude window discriminator (BAK Electronics) to isolate spikes, and then sent to a TEMPO data acquisition system (Reflective Computing), which saved spike time stamps to hard disk along with eye position signals and trial event markers such as stimulus onset/offset. In addition, raw spike waveforms were digitized and recorded to disk using Spike2 software and a Power 1401 data acquisition system (Cambridge Electronic Design). These raw signals were used for extracting MU activity.

Recording protocols. When a well isolated action potential was found, various tuning properties were measured while the animal performed a fixation task. The location and size of the neuron's receptive field were first determined manually using flickering and moving random-dot patterns. Then, tuning curves for direction of motion (eight directions, $45^{\circ}$ apart) and speed of motion (eight speeds, octave steps from $0.5^{\circ} \mathrm{s}$ to $32 \%$, plus $0 \%$ s) were measured quantitatively using coherently moving random-dot patterns. With direction and speed of motion optimized for each neuron, its receptive field was remapped quantitatively to obtain more accurate estimates of receptive field location and size. Tuning for stimulus size (six sizes, octave steps from $1^{\circ}$ to $32^{\circ}$ ) was then measured to determine the optimal stimulus size that elicited the maximum response from the neuron. Finally, we used random-dot stereograms that consisted of a circular center patch and an annular surround to measure tuning for relative disparity. The center patch was placed over the receptive field and had the optimal size for the neuron. The surround patch had a size that was three times the diameter of the center patch. All dots in the random-dot stereogram moved coherently in the optimal direction and speed for the neuron. The horizontal disparity of the center patch was chosen from nine values spaced at $0.4^{\circ}$ intervals between $-1.6^{\circ}$ and $1.6^{\circ}$, whereas the disparity of the surrounding annulus was set at $-0.5^{\circ}$, $0^{\circ}$, or $0.5^{\circ}$. All stimulus conditions were randomly interleaved and repeated at least five times. The animal performed a standard fixation task while visual stimuli were presented for $1.5 \mathrm{~s}$, and the entire experimental protocol generally required at least $40-60 \mathrm{~min}$ to complete per neuron. Because of the length of the protocol and the fact that we maintained fairly strict criteria for SU isolation, many neurons were lost before the entire protocol could be completed.

Data analysis. To analyze SU data, we computed the mean firing rate (over the $1.5 \mathrm{~s}$ stimulus epoch) for each distinct stimulus condition and plotted the data as tuning curves. Disparity-tuning curves were fitted with a Gabor function of the form

$$
R=R_{0}+A \cdot \exp \left\{\frac{-\left(d-d_{0}\right)^{2}}{2 \sigma^{2}}\right\} \cdot \cos \left\{2 \pi f\left(d-d_{0}\right)+\varphi\right\},
$$

where $R$ is the response of the neuron and $d$ denotes stimulus disparity. The free parameters, $R_{0}, A, d_{0}, \sigma, f$, and $\varphi$, correspond to baseline firing rate, response amplitude, center of the Gaussian envelope, Gaussian width, disparity frequency, and disparity phase, respectively. Fitting was performed in MATLAB (MathWorks) using a nonlinear minimization function 'fmincon'. A $\chi^{2}$ goodness-of-fit test was performed to assess the quality of each fit at a significance level $(p)$ of 0.05 , and poor fits were excluded. For additional details of the fitting procedures and the statistical test, see DeAngelis and Uka (2003).

Based on the best-fitting Gabor function, we estimated the neuron's preferred disparity as the disparity at which the maximum response was elicited. Because the range of stimulus disparity was limited, preferred disparity values that exceeded the range were truncated at the limits and were excluded from analysis when their accurate estimates were required (i.e., Fig. $11 A, B$ ). We also computed the disparity discrimination index (DDI) as a measure of the neuron's ability to discriminate disparities based on neural firing rates (Prince et al., 2002b; Watanabe et al., 2002; DeAngelis and Uka, 2003):

$$
D D I=\frac{R_{\max }-R_{\min }}{R_{\max }-R_{\min }+2 \sqrt{\mathrm{SSE} /(N-M)}},
$$

where $R_{\max }$ and $R_{\min }$ represent maximum and minimum responses, respectively, and SSE, $N$, and $M$ denote the sum-squared error, the number of trials, and the number of stimulus conditions, respectively. Disparitytuning (and DDI) was regarded as significant if the tuning curve showed a significant response modulation according to ANOVA $(p<0.05)$.

To examine whether disparity-tuning shifted with the disparity of the surround stimulus in a manner consistent with mechanisms that encode relative disparity, we computed a shift ratio (SR) from a pair of disparitytuning curves measured at two different surround disparities:

$$
S R=\frac{\Delta d_{\mathrm{p}}-\Delta V}{\Delta d_{\mathrm{s}}-\Delta V}
$$


where $\Delta d_{\mathrm{p}}$ denotes the difference in position along the disparity axis between tuning curves measured at the two surround disparities, $\Delta d_{\mathrm{s}}$ indicates the difference between the surround disparities, and $\Delta V$ represents the difference in mean vergence angle between the two surround stimulus conditions (Thomas et al., 2002; Uka and DeAngelis, 2006; Umeda et al., 2007). Note that when $\Delta d_{\mathrm{s}}$ and $\Delta V$ have similar values, the shift ratio could become spuriously large. However, this was never the case in our data; the largest magnitude of $\Delta V$ that we observed was $0.19^{\circ}$, which is substantially smaller than the values of $\Delta d_{\mathrm{s}}\left(0.5^{\circ}\right.$ or $\left.1^{\circ}\right)$. On average, $\Delta V$ was quite small in our experiments $\left(0.005^{\circ} \pm 0.04^{\circ} \mathrm{SD}\right)$, indicating that vergence angle was well matched across surround disparities.

We estimated $\Delta d_{\mathrm{p}}$ using three models: the peak/trough-shift model, the center-shift model, and the phase-shift model. In the peak/troughshift model, it is assumed that the location of a tuning curve along the disparity axis can be represented by its peak or trough, whichever is more pronounced, and the peak or trough location was obtained from an independent Gabor fit to each of the two tuning curves measured at different surround disparities. Then, $\Delta d_{\mathrm{p}}$ was computed as the difference in peak location between the best fit tuning curves if they both had a Gabor phase within $\pm 90^{\circ}$ (both tuning curves have a pronounced peak, like the example shown in Fig. $1 B$ ), the difference in trough location if they both had a Gabor phase larger than $90^{\circ}$ but smaller than $270^{\circ}$ (both tuning curves have a pronounced trough, like the example shown in Fig. $1 E)$, or the difference in either peak or trough location, whichever gives the smaller difference, if one tuning curve has a Gabor phase within $\pm 90^{\circ}$ while the other has a Gabor phase between $90^{\circ}$ and $270^{\circ}$. Most tuning pairs $(126 / 191)$ met the first criterion.

In the center-shift model, it is assumed that the Gaussian center of a fitted Gabor function represents the location of the tuning curve on the disparity axis, and $\Delta d_{\mathrm{p}}$ was computed as the difference in Gaussian center parameters between two tuning curves that were fitted simultaneously by a pair of Gabor functions that shared all parameters except the Gaussian center, amplitude, and baseline response. Previous studies that computed shift ratios for neurons in other visual areas all used the center-shift model (Uka and DeAngelis, 2006; Umeda et al., 2007) or a slight variant in which amplitude and baseline parameters were also shared by the two Gabor functions (Cumming and Parker, 1999; Thomas et al., 2002).

Finally, in the phase-shift model, it is assumed that the Gabor phase angle represents the tuning position, and $\Delta d_{\mathrm{p}}$ was computed as the difference in Gabor phase between two tuning curves that were fitted simultaneously with a pair of Gabor functions that shared all parameters except the Gabor phase, amplitude, and baseline response. Regardless of the model used, a shift ratio of 0 indicates that the location of the tuning curve along the disparity axis did not change depending on the surround disparity (absolute disparity coding), whereas a shift ratio of 1 indicates that the tuning position shifted by the same amount as the change in surround disparity (relative disparity coding). A shift ratio may be negative if the tuning curve shifts in the direction opposite to the change in surround disparity.

For all models, statistical significance of shift ratios was assessed by bootstrap analysis (Efron and Tibshirani, 1993). Responses to each center disparity were resampled randomly with replacement (same number of trials as in the original data) to obtain a simulated tuning curve for each surround disparity. Each pair of simulated tuning curves for two particular surround disparities was processed in exactly the same manner as the measured tuning curves to compute a bootstrap shift ratio. By repeating this process 400 times, we obtained a distribution of bootstrap shift ratios. A measured shift ratio was considered significantly greater than 0 if the proportion $(p)$ of bootstrap shift ratios smaller than 0 was $<5 \%(p<0.05)$. Similarly, a measured shift ratio was considered significantly smaller than 0 if $<5 \%$ of the bootstrap shift ratios were positive.

We also used the results of the bootstrap analysis to assess whether or not the response amplitude at the preferred disparity changed with surround disparity. We took the ratio of the response at the preferred disparity in one surround condition to that in another surround condition (amplitude ratio) for each pair of simulated tuning curves and obtained a distribution of bootstrap amplitude ratios. A response amplitude ratio was considered significantly greater than 1 if the proportion $(p)$ of boot- strap amplitude ratios smaller than 1 was $<5 \%(p<0.05)$. Similarly, an amplitude ratio was considered significantly smaller than 1 if $<5 \%$ of the bootstrap amplitude ratios were $>1$.

To test for clustering of disparity-tuning properties, we extracted MU responses offline and analyzed them in the same manner as we did for the SU data. MU responses were extracted from the raw spike waveforms collected using Spike2 software (CED) by setting a spike detection threshold (amplitude threshold) such that the spontaneous rate for MU activity was $\sim 75$ spikes/s higher than the spontaneous rate for SU activity. To make the MU signal independent of the SU activity, each SU spike was removed (offline) from the MU activity. The success of this manipulation was confirmed by computing cross-correlograms between the SU and MU spike trains, both before and after SU spikes were removed from the MU signal (see Chen et al., 2008 for details).

Identification of cortical areas. Using sMRI maps that have been registered with a surface-based atlas of macaque visual areas (Van Essen et al., 2001) as a guide, we characterized responses of neurons at dozens of locations within the recording grid to identify visual cortical areas along each electrode penetration. Depths of gray and white matter transitions were noted, and receptive field locations and sizes as well as basic response properties, such as direction, speed, and disparity selectivity, were determined qualitatively. Based on how receptive field location and size changed within electrode penetrations and across locations in the recording grid, the locations and depths of areas V3 and V3A and their neighboring areas were estimated. Subsequently, electrode penetrations were focused on those tracks that passed through the estimated locations of V3 and V3A, and SU data were collected in these areas. Receptive field locations and sizes measured quantitatively for the recorded units were then incorporated into the area identification process to help ensure that the correct area was assigned to each neuron. The portion of area V3 that we recorded from (V3 dorsal) only contains neurons with receptive fields in the lower quadrant of the visual field, while area V3A contains neurons with receptive fields in either the upper or lower quadrants depending on the location within V3A (Van Essen and Zeki, 1978; Zeki, 1978; Gattass et al., 1988). Most recordings made in V3A were from neurons with clear upper visual field representations so that they could be easily distinguished from V3 neurons. We included recordings from V3A neurons with lower visual field representations only when we were confident that they were indeed in V3A. We combined V3A data from the upper and lower visual fields, since we did not observe any clear changes in our results when V3A data were restricted to the lower visual field. When receptive field locations were on either the horizontal meridian (the V2-V3 border) or the vertical meridian (the V3-V3A border), we compared their receptive field sizes with published data for given eccentricities (for a summary of published data, see Smith et al., 2001), and neurons were excluded if their receptive fields sizes did not indicate clearly to which area they belonged.

\section{Results}

We recorded from $155 \mathrm{~V} 3$ and 412 V3A neurons in two monkeys. Of these, we successfully completed our experimental protocol for a total of $78 \mathrm{~V} 3$ (monkey R: 53; monkey P: 25) and $174 \mathrm{~V} 3 \mathrm{~A}$ neurons (monkey R: 65; monkey P: 109), which are analyzed further here. We ran our experimental protocol on virtually every neuron we encountered and continued data collection as long as we could maintain good isolation of the neuron's action potential and the animal continued to perform the fixation task. There was no selection of neurons at any step of the protocol based on tuning properties, except that we sometimes abandoned a neuron if it did not respond well to any of our stimuli. We never abandoned a responsive neuron, even if it did not show tuning in our stimulus protocols. Therefore, as far as we could control, there was no experimenter bias in sampling neurons.

We first present example disparity-tuning functions and compare the basic tuning characteristics of a population of neurons with previous results from other cortical areas. Then, we examine whether V3 and V3A neurons are selective for relative disparity. 

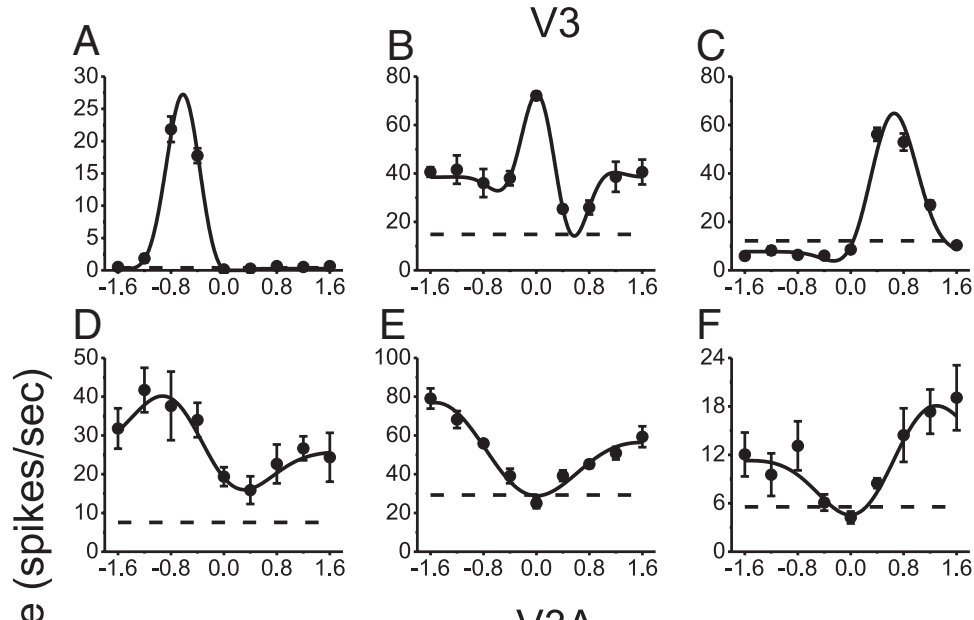

\section{$\mathrm{F}$}
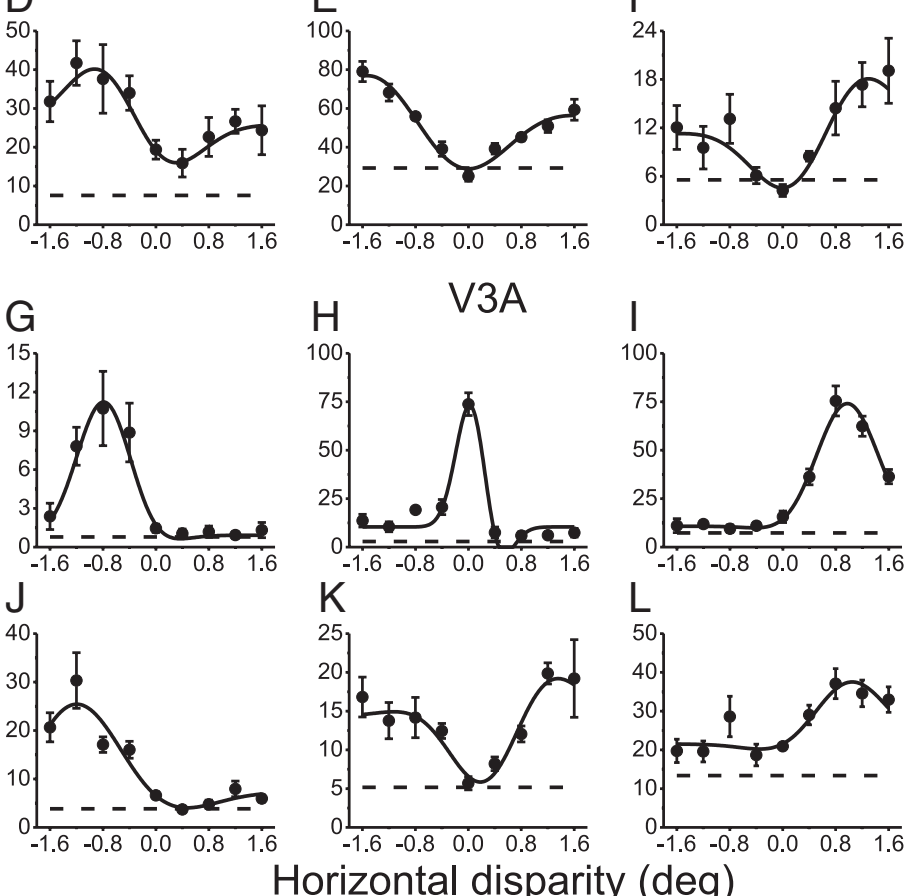

Horizontal disparity (deg)

Figure 1. Horizontal disparity-tuning curves for six example neurons from area V3 $(\boldsymbol{A}-\boldsymbol{F})$ and six neurons fromV3A $(\boldsymbol{G}-\boldsymbol{L})$. In each case, disparity of the center patch was varied while the surround disparity remained 0 (fixation plane). Solid lines are the Gabor functions that best fit the data. Horizontal dashed lines indicate spontaneous activity levels. Each error bar indicates the SEM. deg, Degrees $\left({ }^{\circ}\right)$.

Finally, we compare SU and MU tuning properties to see whether neurons with similar disparity-tuning characteristics are clustered in $\mathrm{V} 3$ and $\mathrm{V} 3 \mathrm{~A}$.

\section{Examples of disparity-tuning in V3 and V3A}

Many of the neurons in V3 and V3A that we examined were tuned to binocular disparity, and the shape of the tuning curve varied from neuron to neuron. Figure 1 shows examples of disparity-tuning for several neurons; in each case, the disparity of the center stimulus was varied while the disparity of the surround was fixed at 0 (fixation plane). Some neurons were narrowly tuned to near disparities (Fig. $1 A, G$ ), zero disparity (Fig. $1 B, H$ ), or far disparities (Fig. $1 C, I$ ), while others were more broadly tuned (Fig. $1 D, F, J, L$ ). There were also neurons that responded more or less equally to near and far disparities but gave weak responses to zero disparity (Fig. $E, K$ ). These tuning functions are qualitatively comparable to the six types of disparity-tuning reported by Poggio et al. (1988), although we have no evidence that these form distinct classes in our data.

\section{Population summary of disparity selectivity}

To summarize disparity selectivity across the populations of neurons recorded from V3 and V3A and to compare our results with those from other cortical areas published previously, we computed a disparity discrimination index (DDI) for each tuning

curve. This index provides a measure of how well the neuron can discriminate between its preferred and null disparities relative to its intrinsic response variability, and the index ranges from 0 (indiscriminable) to 1 (perfectly discriminable). Figure 2, $A$ and $B$, shows histograms of DDI for populations of neurons recorded from $\mathrm{V} 3$ and V3A, respectively. Of $78 \mathrm{~V} 3$ neurons and $174 \mathrm{~V} 3 \mathrm{~A}$ neurons that we examined quantitatively, 56\% (44/78) of V3 and $53 \%(93 / 174)$ of $\mathrm{V} 3 \mathrm{~A}$ neurons showed significant tuning (ANOVA, $p<$ 0.05 , filled bars). These values are roughly comparable to the proportion of V3 neurons selective for binocular disparity of bar stimuli in anesthetized monkeys (Felleman and Van Essen, 1987; Adams and Zeki, 2001) (but see Poggio et al., 1988, for alert fixating monkey data). The mean value of DDI for V3 neurons is 0.54 , while the mean DDI for $\mathrm{V} 3 \mathrm{~A}$ neurons is 0.51 ; these two values are not significantly different ( $t$ test, $p=0.24$ ).

If $\mathrm{V} 3$ and $\mathrm{V} 3 \mathrm{~A}$ play privileged roles in stereoscopic depth perception, as has been suggested by some fMRI studies (e.g., Tsao et al., 2003), one might expect that disparity selectivity in these areas would be significantly stronger than in other areas. To examine this possibility, we compared population distributions of DDIs from various cortical areas. The DDI histograms in Figure $2 A, B$ are replotted in Figure $2 C$ as cumulative distributions, together with corresponding data from V1 (Prince et al., 2002b), V4 (Tanabe et al., 2005), and MT (DeAngelis and Uka, 2003) for comparison. The distributions of DDIs for V1 (blue), V3 (red), V3A (cyan), and V4 (pink) are quite similar. In contrast, the MT distribution (green) is clearly shifted toward substantially higher DDI values, indicating a greater incidence of neurons with strong disparity selectivity. Therefore, with respect to this measure of disparity discriminability, areas V3 and V3A are no more sensitive to binocular disparity than any of the early and mid-level visual areas that we considered.

\section{Disparity-tuning characteristics}

To quantitatively summarize disparity-tuning characteristics, we fitted a Gabor function to the tuning curve of each neuron that showed a significant response variation with disparity (ANOVA, $p<0.05$ ). From these fits, we extracted some key parameters that capture basic features of disparity-tuning, including the preferred disparity, Gabor phase, and Gabor frequency. The solid curves in Figure 1 indicate the best-fitting Gabor functions for each of the example neurons. A $\chi^{2}$ goodness-of-fit test was used to exclude neurons with poor fits at a significance level $(p)$ of 0.05 , and tuning parameters extracted from the acceptable fits for $39 \mathrm{~V} 3$ neurons and $84 \mathrm{~V} 3 \mathrm{~A}$ neurons are summarized here. Figure 3, $\mathrm{A}$ and $B$, shows histograms of preferred disparities for $\mathrm{V} 3$ and $V 3 \mathrm{~A}$, respectively. In $\mathrm{V} 3$, the distribution had a median of $-0.09^{\circ}$, which is not significantly different from 0 (signed-rank test, 

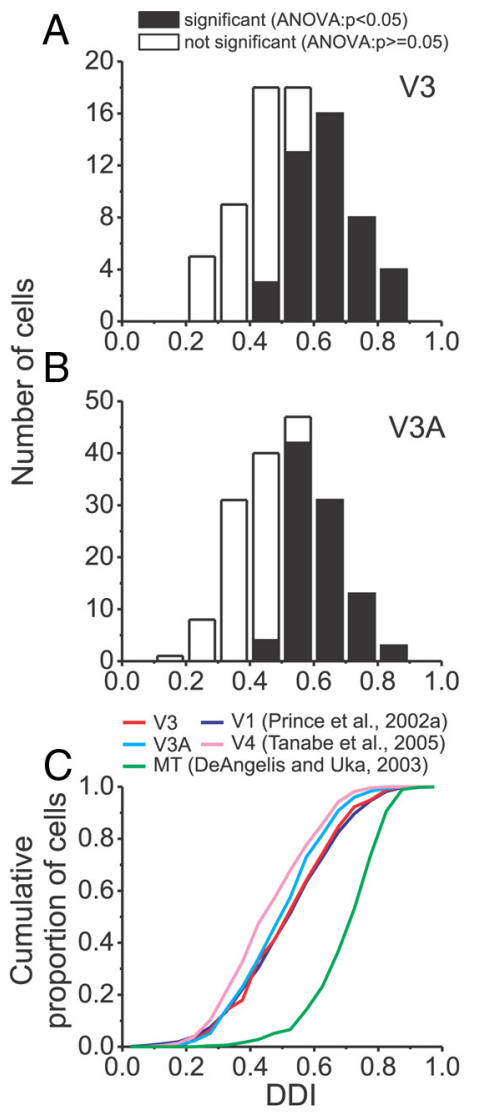

Figure 2. Population distributions of the disparity discrimination index. $A, D D I$ distribution for V3 neurons. Black bars indicate neurons with significant disparity-tuning (ANOVA, $p<$ 0.05). B, DDI distribution for V3A neurons; format as in $A$. C, Cumulative distributions of DDIs for V3 (red) and V3A (cyan) neurons, together with corresponding data for V1 (blue), V4 (pink), and MT (green) neurons. The V1, V4, and MT data are replotted from Prince et al. (2002b), Tanabe et al. (2005), and DeAngelis and Uka (2003), respectively.

$p=0.37)$. The $\mathrm{V} 3 \mathrm{~A}$ distribution had a median $\left(-0.26^{\circ}\right)$ that was also not significantly different from 0 (signed-rank test, $p=0.83$ ).

Previous studies reported that distributions of disparity preferences in V4 (Tanabe et al., 2005) and MT (DeAngelis and Uka, 2003) have a bias toward near disparities (see also Adams and Zeki, 2001). However, our results in V3 and V3A did not show any obvious bias. When the disparity preference distributions are compared across cortical areas in the form of cumulative distributions (Fig. 3C), the V3 (red) and V3A (cyan) distributions are similar to those of V4 (pink) and MT (green), except that the latter areas show the aforementioned bias toward near disparities. The only area that shows a markedly different distribution of disparity preferences is V1. The V1 distribution (blue) has a clearly steeper slope than the other distributions, which reflects the narrow range of disparity preferences exhibited by V1 neurons. Although the V1 data were obtained from a narrower range of eccentricities (the central $6^{\circ}$ ) than data from the other areas (up to $\sim 30^{\circ}$ ), it is unlikely that eccentricity can account for differences in the distributions of disparity preference between areas (Fig. 3C) for two reasons. First, the range of preferred disparities for $\mathrm{V} 1$ neurons having larger eccentricities $\left(8-22^{\circ}\right)$ is similar to that shown in Fig. 3C (Durand et al., 2002). Second, limiting our $\mathrm{V} 3$ and V3A data to neurons with eccentricities $<10^{\circ}$ yielded similar distributions of preferred disparities (Fig. 3C, dashed lines). This is also consistent with the fact that we found little or no correlation between the magnitude of preferred disparity and
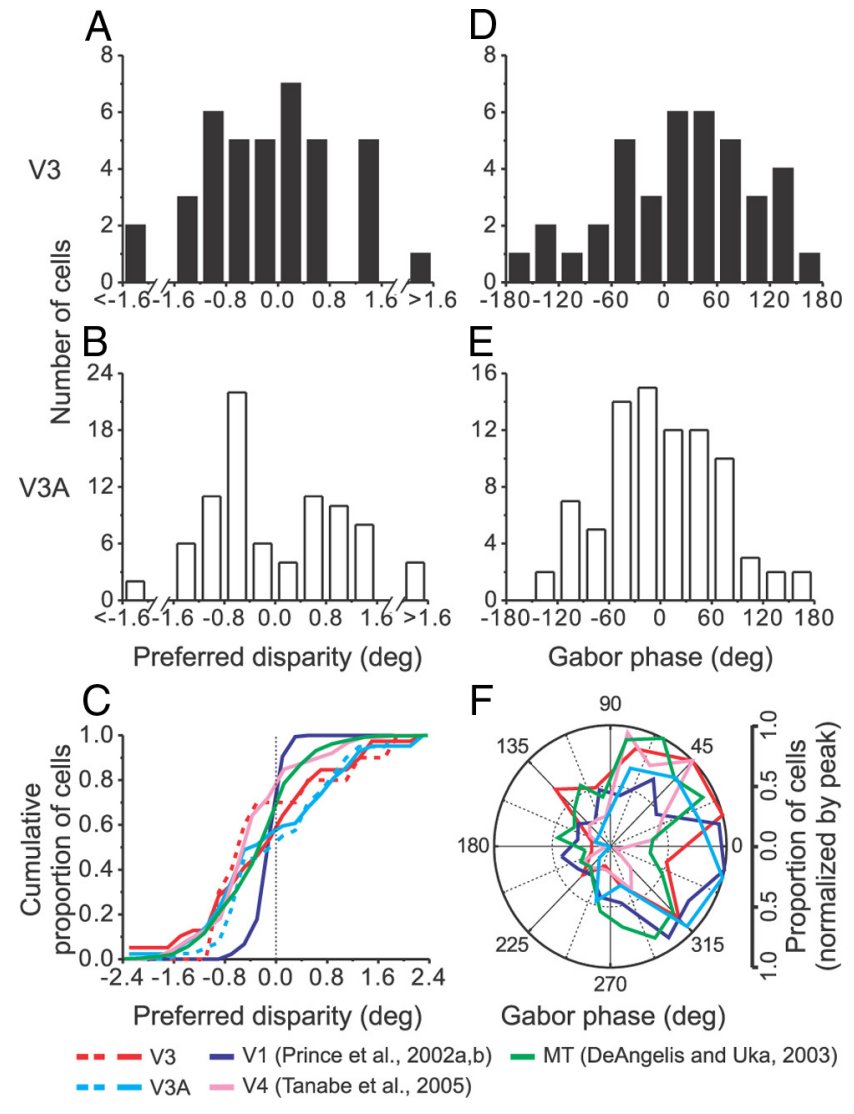

Figure 3. Population distributions of preferred disparity $(\boldsymbol{A}-\boldsymbol{C})$ and Gabor phase $(\boldsymbol{D}-\boldsymbol{F})$. $\boldsymbol{A}$ The distribution of preferred disparities for $\mathrm{V} 3$ neurons. $\boldsymbol{B}$, The distribution of preferred disparities for V3A neurons. C, Cumulative distributions of preferred disparities for V3 (red) and V3A (cyan) neurons and corresponding data for V1 (blue), V4 (pink), and MT (green) neurons, which are replotted from Prince et al. (2002b), Tanabe et al. (2005), and DeAngelis and Uka (2003), respectively. Dashed lines indicate distributions for neurons with eccentricities $\angle 10^{\circ}$. $\boldsymbol{D}$, The distribution of Gabor phase for V3 neurons. $\boldsymbol{E}$, The distribution of Gabor phase for V3A neurons. $\boldsymbol{F}$, Distributions of Gabor phase for V3 and V3A neurons, together with data for V1, V4, and MT neurons that are replotted from the previous studies cited above except for V1 data, which are from Prince et al. (2002a). Color conventions as in C. deg, Degrees $\left({ }^{\circ}\right)$.

eccentricity (V3: $r=0.048, p=0.77$; V3A: $r=0.25, p=0.02$ ), as was also found previously for V4 (Tanabe et al., 2005) and MT (DeAngelis and Uka, 2003).

Figure 3, $D$ and $E$, shows distributions of the Gabor phase parameter for V3 and V3A, respectively. The Gabor phase describes the shape of the tuning curve; phases near $0^{\circ}$ and $\pm 180^{\circ}$ indicate disparity-tuning curves that are approximately even symmetric, whereas phases near $90^{\circ}$ and $270^{\circ}$ represent oddsymmetric tuning (e.g., $90^{\circ}$ indicates a peak for near disparities and a trough for far disparities). In both V3 and V3A, phase distributions are broadly distributed around 0 . Circular means of the distributions are $36.3^{\circ}$ and $-0.05^{\circ}$ for $\mathrm{V} 3$ and $\mathrm{V} 3 \mathrm{~A}$, respectively, and these values are not significantly different from 0 (test for circular mean, $p>0.05$ ).

Previously, it has been reported that the Gabor phase distribution for V1 neurons is unimodal around 0 (Prince et al., 2002a), whereas the phase distributions for V4 neurons (Tanabe et al., 2005) and MT neurons (DeAngelis and Uka, 2003) are bimodal with peaks around $\pm 45^{\circ}-60^{\circ}$. In Figure $3 F$, phase histograms from $D$ and $E$ are replotted on polar coordinates along with the phase distributions from V1 (Prince et al., 2002a), V4 (Tanabe et al., 2005), and MT (DeAngelis and Uka, 2003). The V3A distribution (cyan) is quite similar to that of V1 (blue), 

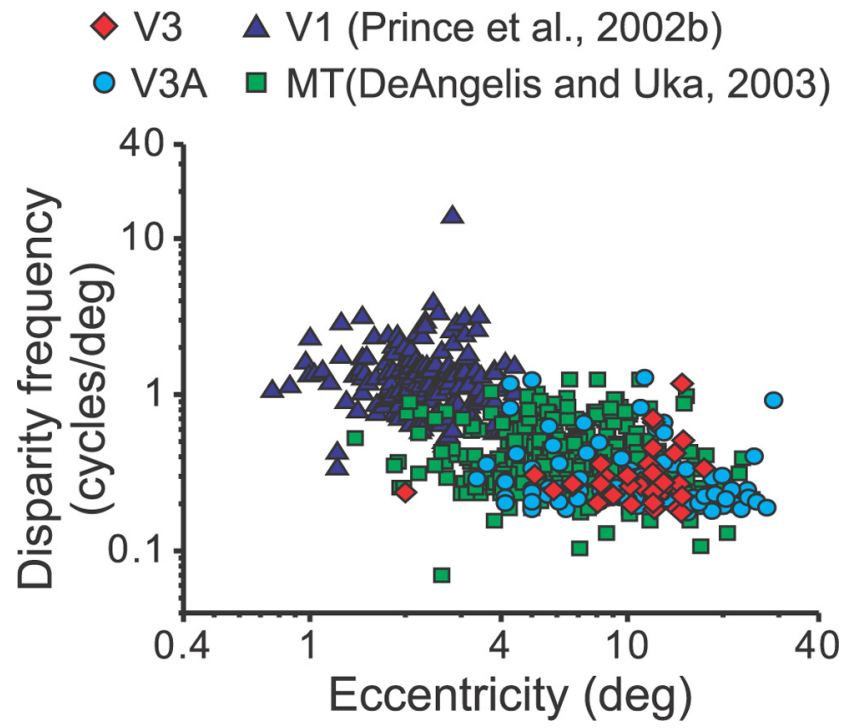

Figure 4. Disparity frequency as a function of eccentricity for V3 (red diamonds) and V3A (cyan circles) neurons. For comparison, data for V1 (blue triangles) and MT (green squares) neurons are replotted from Prince et al. (2002a) and DeAngelis and Uka (2003), respectively.

indicating a broad tendency toward even-symmetric disparitytuning. By comparison, the V3 distribution (red) shows a hint of bifurcation, suggesting a bias toward asymmetric tuning although it is weaker than that seen in V4 (pink) and MT (green) and is not statistically significant (mode test, $p>0.05$; based on Fisher and Marron, 2001).

Another parameter of interest is the frequency of the Gabor function, or "disparity frequency," It largely determines how quickly responses taper off as the stimulus disparity moves away from the neuron's preferred disparity (provided that the Gaussian envelope tapers off more slowly than the sinusoidal component of the Gabor function), and it is inversely related to the width of disparity-tuning. Thus, it gives a measure of disparity resolution, analogous to the preferred spatial frequency of V1 receptive fields. Just as spatial frequency preference varies with receptive field eccentricity in V1 (e.g., Schiller et al., 1976; Movshon et al., 1978), the disparity frequency may also be expected to change with eccentricity (Prince et al., 2002a; DeAngelis and Uka, 2003) (but see Tanabe et al., 2005). Figure 4 shows disparity frequencies of V3 and V3A neurons as a function of eccentricity, along with corresponding data from V1 (Prince et al., 2002a) and MT (DeAngelis and Uka, 2003). At comparable eccentricities, disparity frequencies are substantially higher (i.e., disparity-tuning is narrower) for V1 (blue) neurons than MT (green) neurons. Our data from V3 (red) and V3A (cyan) overlap extensively with those from MT. This suggests that, in areas beyond V1, there may be a substantial pooling of neurons in the disparity domain.

In summary, disparity-tuning curves of neurons in V3 and V3A are not particularly distinct from those of other early and mid-level visual areas with respect to selectivity, disparity preference, and the shape of disparity-tuning. This does not mean that V3 and V3A do not play important roles in stereoscopic depth processing. For instance, these areas might encode relative disparities, which are thought to be important for fine depth judgments. Next, we examine whether neurons in V3 and V3A are primarily selective for relative or absolute disparity.
Relative disparity selectivity: disparity-tuning as a function of surround disparity

The visual system is thought to compute relative disparity and use it for fine depth discrimination (Westheimer, 1979). If neurons encode the relative disparity between center and surround stimuli, then their disparity-tuning for the center stimulus should shift with the disparity of the surround such that response depends on the difference in disparity between center and surround stimuli. This prediction was tested previously in areas V1, V2, V4, and MT (Cumming and Parker, 1999; Thomas et al., 2002; Uka and DeAngelis, 2006; Umeda et al., 2007). We used the same stimulus configuration and similar analysis methods as those in the previous studies to examine the relative disparity-tuning of neurons in $\mathrm{V} 3$ and V3A.

Figure 5 shows examples of disparity-tuning for $\mathrm{V} 3$ ( $A, C, E$, $G)$ and V3A $(B, D, F, H)$ neurons measured at three different surround disparities. For many neurons, disparity-tuning for the center stimulus was little affected by surround disparity (Fig. $5 A, B)$, indicating that these neurons are selective for absolute, not relative, disparity. When the surround disparity did affect tuning for the center stimulus, we most typically observed changes in the amplitude and/or baseline response level of the tuning curve. For example, the disparity-tuning functions shown in Figure 5, $C$ and $D$, mainly changed their amplitudes depending on the surround disparity. The examples shown in Figure 5, E and $F$, illustrate how baseline response may change with surround disparity, in addition to changes in amplitude. We found that 40\% of neurons (V3: 44\%; V3A: 38\%) showed significant changes in response amplitude with surround disparity (bootstrap analysis, $p<0.05$; see Materials and Methods). One might wonder whether surround disparity-dependent modulations of response amplitude could serve to represent relative disparity. Indeed, neurons that exhibit such modulations would be maximally responsive to a particular combination of center and surround disparities. However, their responses would not be consistently tuned to a particular difference between center and surround disparities (i.e., relative disparity) independent of the absolute disparities of center and surround. Therefore, although amplitude-modulated neurons may carry information necessary to extract relative disparity at later stages of processing, they do not explicitly represent relative disparity. In contrast, neurons with a disparity-tuning curve that shifts horizontally with surround disparity, such as those shown in Figure 5, $G$ and $H$, could at least partially signal relative disparity.

To quantify whether disparity-tuning curves shifted with surround disparity in a manner that is consistent with relative disparity encoding, we made pairwise comparisons between disparity-tuning functions for all distinct pairings of the three surround disparities that were tested for each neuron. Because tuning amplitude and baseline response often depended on surround disparity, we first compared DDIs between pairs of surround disparities to exclude cases in which one tuning curve did not show significant tuning. Figure $6, A$ and $B$, shows scatter plots of the DDI for one surround disparity versus another for each pair of tuning curves obtained from V3 and V3A neurons, respectively. Although DDI values were strongly correlated across surround disparities overall (V3: $r=0.59, p<0.01$; V3A: $r=0.62$, $p<0.01$ ), disparity-tuning functions of some neurons were weakened by certain surround disparities, resulting in nonsignificant DDI values (Fig. 6, gray triangles). Of 234 pairs of tuning curves from V3 and 522 pairs from V3A, 91 pairs from $41 \mathrm{~V} 3$ neurons and 184 pairs from $90 \mathrm{~V} 3 \mathrm{~A}$ neurons had significant DDIs at both surround disparities and were analyzed further. 
V3

V3A

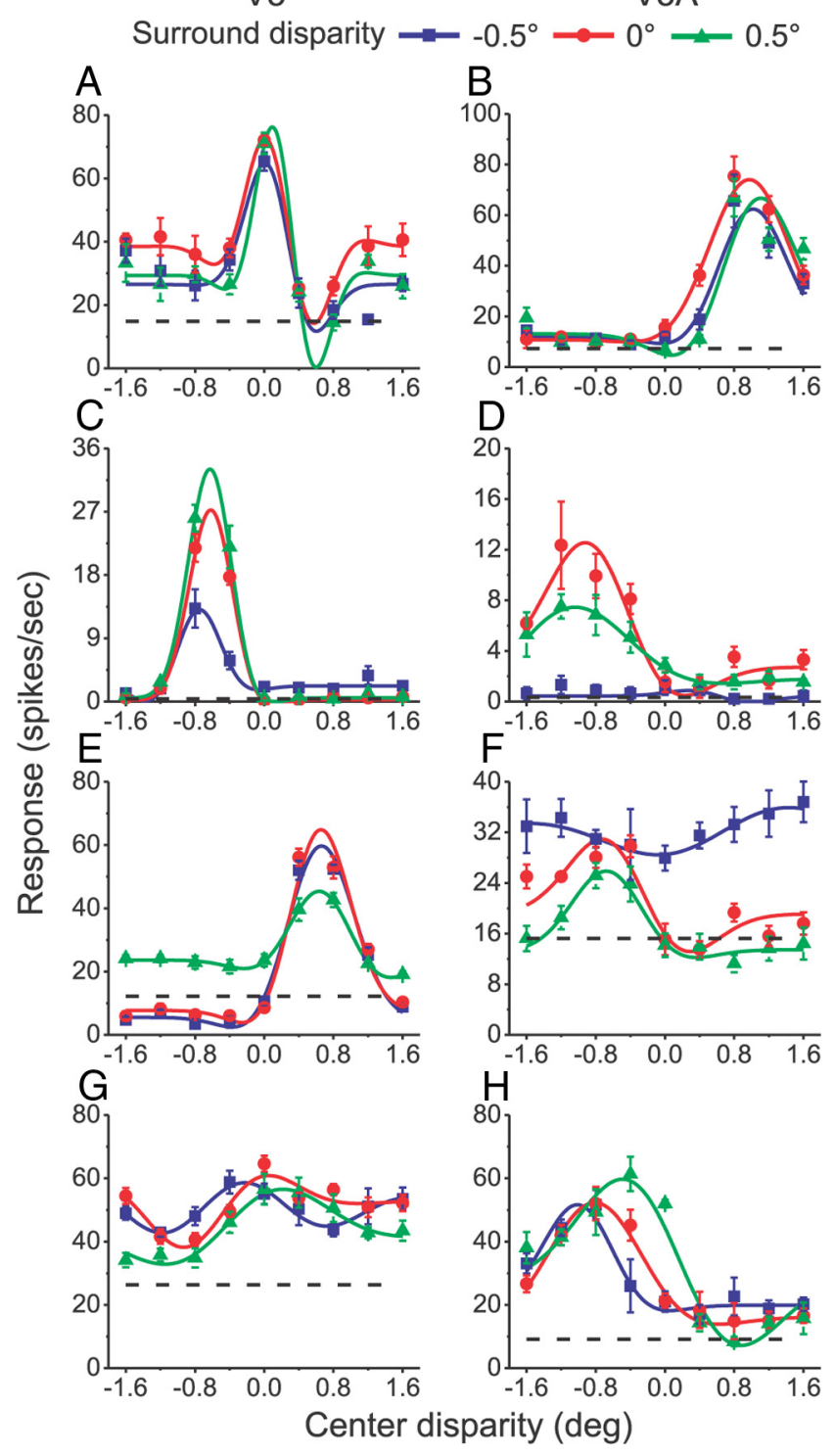

Figure 5. Examples of disparity tuning as a function of surround disparity $\left(-0.5^{\circ}, 0^{\circ}\right.$, and $0.5^{\circ}$ shown in blue, red, and green, respectively) from $\mathrm{V} 3$ neurons $(\boldsymbol{A}, \boldsymbol{C}, \boldsymbol{E}$, and $\boldsymbol{G})$ and $\mathrm{V} 3 \mathrm{~A}$ neurons $(\boldsymbol{G}, \boldsymbol{D}, \boldsymbol{F}$, and $\boldsymbol{H})$. Solid lines indicate Gabor functions that best fit the data. Horizontal dashed lines indicate spontaneous activity levels. Each error bar indicates the SEM. $\boldsymbol{A}$, The tuned-zero V3 neuron shown in Figure $1 B$. B , The tuned-far V3A neuron of Figure 1 I. C, The tuned-near $V 3$ neuron from Figure $1 A$, which shows amplitude changes with surround disparity. $\boldsymbol{D}, A$ V 3 A neuron showing flattening of tuning for the near surround disparity. $E$, The tunedfar V 3 neuron of Figure $1 C$, showing changes in baseline response. $\boldsymbol{F}$, A tuned-near V3A neuron showing flattening of tuning at an elevated baseline. $G, A$ V3 neuron with some degree of tuning shift with surround disparity. $\boldsymbol{H}, \mathrm{A}$ V3A neuron with some degree of peak shifting. deg, Degrees $\left({ }^{\circ}\right)$.

For each of the remaining pairs of tuning curves, the horizontal shift between them was divided by the difference between the surround disparities, to obtain a "shift ratio" (after corrections for small differences in vergence angle between surround disparity conditions; see Materials and Methods). A shift ratio of 0 indicates no shift in disparity-tuning depending on surround disparity and is consistent with coding of absolute disparity, whereas a shift ratio of 1 indicates a shift in tuning equal to the change in surround disparity and is consistent with coding of relative disparity. Note that a shift ratio can be negative if the tuning curve shifts in the direction opposite to the change in surround disparity.
- both DDI significant

$\Delta$ one DDI significant

A $\square$ neither DDI significant

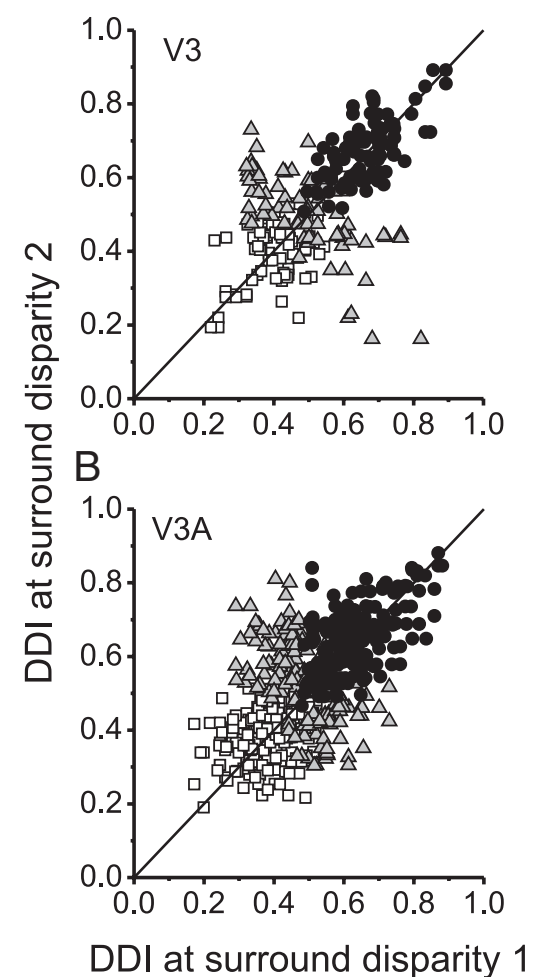

Figure 6. Comparison of $\mathrm{DDl}$ values for pairs of disparity-tuning curves measured at different surround disparities. Black circles, Cases where disparity-tuning is significant for both surround disparities; gray triangles, cases where tuning is significant for one of the two surround disparities; white squares, cases where neither surround disparity has significant tuning. $A$, Data from area V3. B, Data from V3A.

Shift ratios were computed using three models, as described in Materials and Methods. In the peak/trough-shift model, it is assumed that the location of the tuning peak (or trough) shifts with surround disparity, and a shift ratio was computed based on independent Gabor fits to each pair of tuning curves. Figure 7, A and $B$, shows histograms of shift ratios obtained from the peak/ trough-shift model for populations of V3 and V3A neurons, respectively. The shift ratios are distributed roughly around 0 with median values of 0.05 and 0.07 for V3 and V3A, respectively, which are significantly greater than 0 (signed-rank test, $p=0.025$ for V3 and $p<0.01$ for V3A). However, only a few individual shift ratios were significantly greater than 0 (bootstrap analysis, $p<0.05)$ (Fig. $7 A, B$, filled bars). We also computed shift ratios based on a spline fit to each tuning curve and obtained similar but somewhat broader shift ratio distributions (data not shown; median shift ratios: 0.04 and 0.06 for V3 and V3A, respectively, neither of which is significantly different from 0 based on signedrank test, $p>0.05$ ). These shift ratio distributions are largely consistent with absolute disparity coding, although there is a slight overall bias toward relative disparity coding. For a few pairs of surround disparities, some neurons did show shift ratios near 1 , such that they could be signaling relative disparity. However, none of these large shift ratios in Figure 7, $A$ and $B$, were statistically significantly greater than 0 (bootstrap, $p>0.05$ ), and there are a roughly comparable number of pairs that showed negative shift ratios of similar magnitudes. Therefore, neurons with shift ratios near 1 most likely belong to a tail of a single population 


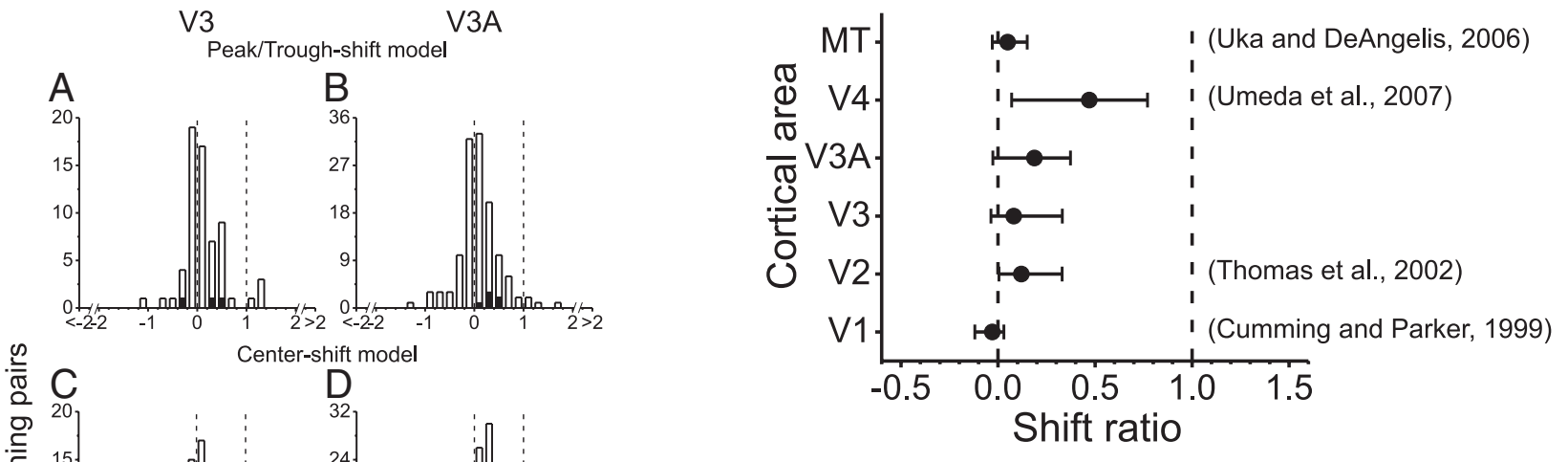

Figure 8. Comparison of shift ratios across various cortical areas. Median shift ratios (based on the center-shift model or a slight variant; see Materials and Methods) for V1 (Cumming and Parker, 1999), V2 (Thomas et al., 2002), V3, V3A, V4 (Umeda et al., 2007), and MT (Uka and DeAngelis, 2006) are plotted (filled circles) along with the interquartile ranges (horizontal error bars). Dashed lines indicate shift ratios of 0 and 1 , which correspond to coding of absolute and relative disparity, respectively.

may cause the center-shift model to overestimate the shift of the tuning curves.

Another possibility is that the disparity-tuning profile shifts due to a change in the shape of the tuning curve with surround disparity, which could be captured by changes in the phase of the best-fitting Gabor function. In this phase-shift model, the Gabor phase angle changes with surround disparity without changing the location of the Gaussian envelope, and a pair of tuning curves were fitted by two Gabor functions that shared all parameters except the Gabor phase, amplitude, and baseline responses. Figure 7, $E$ and $F$, shows distributions of shift ratios for $\mathrm{V} 3$ and V3A, respectively, as obtained from the phase-shift model. Again, most values are closer to 0 than 1 , with median shift ratios of 0.21 and 0.26 for $\mathrm{V} 3$ and $\mathrm{V} 3 \mathrm{~A}$, respectively, which are both significantly greater than 0 (signed-rank test, $p<0.01$ ). As was the case for the center-shift model, we found shift ratios that are significantly greater than 0 as well as those that are significantly smaller than 0 (Fig. $7 E, F$, filled bars). Overall, shift ratio distributions from the phase-shift model also suggest that most neurons in V3 and V3A signal absolute disparity, although there is a bias toward relative disparity coding on average. The larger overall shift ratios for the phase-shift model, relative to the peak/trough-shift model, partly reflect how the shift ratio was computed for this model. The difference in phase of the Gabor fit between a pair of surround disparities was converted to a difference in disparity (by dividing by $2 \pi f$; see Equation 1 ). That is, the phase shift corresponds to the shift of the sinusoidal component between two Gabor fits. However, the fixed Gaussian envelope pulls the peak of the tuning curve toward the center of the envelope, compared to the peak of the sinusoidal component, and thus reduces the actual shift in disparity preference corresponding to a given phase shift. This causes the phase-shift model to overestimate the shift between a pair of tuning curves.

To summarize, Figure 8 shows median shift ratios (filled circles), along with error bars that indicate the range from the 25 th to 75th percentiles, for populations of neurons that we recorded in V3 and V3A as well as other published results from areas V1, V2, V4, and MT (Cumming and Parker, 1999; Thomas et al., 2002; Uka and DeAngelis, 2006; Umeda et al., 2007). Previously, shift ratios for V1 and MT neurons were shown to be narrowly distributed with means near 0 , and therefore consistent with the prediction of absolute disparity coding (see Cumming and Parker, 1999, their Fig. 11C, and Uka and DeAngelis, 2006, their 
Fig. 8C). In contrast, the distribution of shift ratios for V4 neurons is shifted and slightly skewed toward a value of 1 , with a median value near 0.5 (see Umeda et al., 2007, their Fig. 7A). Therefore, responses of a substantial number of V4 neurons are at least partially consistent with coding of relative disparity. The distribution for V2 neurons shows a slight positive skew that extends one tail toward relative disparity coding (Thomas et al., 2002, Fig. $6 B$ ), but the median shift ratio is rather close to 0 . Our data from V3 and V3A neurons are most similar to the V2 data, both in terms of the median shift ratio and the interquartile range, but appear less skewed toward a value of 1 (see Fig. 7) compared to the V2 distribution. Overall, the data suggest strongly that areas V3 and V3A are not particularly specialized to represent relative disparities of center-surround stimuli in comparison to other visual areas.

\section{Comparisons between $\mathrm{SU}$ and $\mathrm{MU}$ responses}

It is well known that neighboring visual cortical neurons often share similar response properties and may be organized into a columnar structure (e.g., Hubel and Wiesel, 1962, 1963). Since such an organization would constrain afferent projections and local circuitry (Mountcastle, 1997), it is useful to know the stimulus dimensions along which neurons are organized to understand neural computations performed by local circuits. Previously, Adams and Zeki (2001) showed that neurons with similar disparity tuning are clustered in V3 and are organized into columns. However, their analysis did not include any quantitative evaluations of tuning similarity. To our knowledge, nothing is known about the organization of disparityselective neurons in V3A.

Here, we examine whether nearby neurons share similar disparity-tuning properties by comparing the disparity-tuning of SU responses with the tuning of simultaneously recorded MU activity at each recording site in V3 and V3A. The SU spikes were subtracted from the MU event train (see Materials and Methods); thus, MU responses reflect the aggregate activity of other neurons in close proximity to the SU. If neighboring neurons all have disparity-tuning properties similar to those of the SU, then MU disparity-tuning should be very similar to that of the SU. On the other hand, if neighboring neurons have diverse disparity-tuning properties, then MU tuning should be substantially broader than SU tuning or may even be flat.

Figure 9 shows examples of MU (solid curves) and SU (dashed curves) disparity-tuning curves measured in $\mathrm{V} 3(A, C, E$, and $G)$ and $\mathrm{V} 3 \mathrm{~A}(B, D, F$, and $H)$ at three different surround disparities. We found that MU tuning was generally similar to SU tuning; both peaked at roughly comparable disparities, and the MU tuning width was either similar to or slightly broader than that of the SU (Fig. 9A-F). The effects of surround disparity were also generally similar between SU and MU tuning. When there was little change in SU tuning as a function of surround disparity, MU tuning was also little affected by the surround (Fig. 9A,B). When amplitude and baseline changes were seen in SU tuning, similar changes were generally observed in the corresponding MU responses (Fig. 9C-E). Figure $9 F$ shows an example in which the peak of MU tuning shifted with surround disparity in a manner roughly similar to that for the SU responses. These examples suggest that neurons with similar disparity-tuning are clustered in both $\mathrm{V} 3$ and $\mathrm{V} 3 \mathrm{~A}$, in agreement with the previous finding by Adams and Zeki (2001) for V3. However, we also found a small number of cases for which SU and MU tuning did not match (Fig. $9 G, H)$. Figure $9 G$ shows an example in which SU and MU responses were tuned to markedly different disparities; MU re-

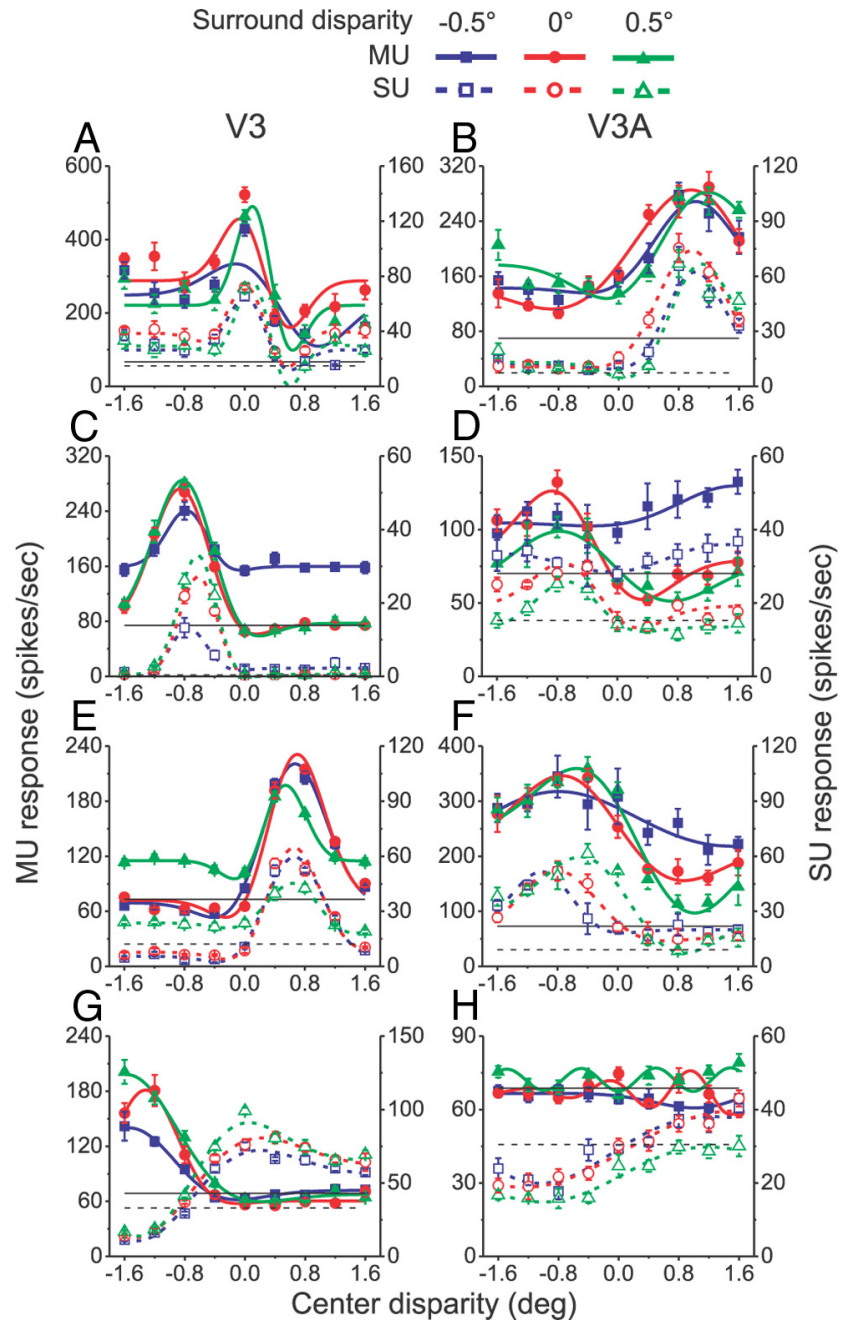

Figure 9. Examples of MU (filled symbols) and SU (open symbols) tuning measured at $-0.5^{\circ}$ (blue), $0^{\circ}$ (red), and $0.5^{\circ}$ (green) surround disparities for four recording sites in V3 $(\boldsymbol{A}, \boldsymbol{C}$, $\boldsymbol{E}, \boldsymbol{G})$ and four additional sites in $\mathrm{V} 3 \mathrm{~A}(\boldsymbol{B}, \boldsymbol{D}, \boldsymbol{F}, \boldsymbol{H})$. Solid and dashed curves are Gabor functions that best fit the MU and SU data, respectively. Solid and dashed horizontal lines indicate spontaneous activity levels for MU and SU responses, respectively. Each error bar indicates the SEM. deg, Degrees $\left({ }^{\circ}\right)$.

sponses were only present for near disparities, whereas SU responses were mostly restricted to far disparities. Recordings such as these may have been made at a boundary between two disparity clusters, although it is also feasible that neurons tuned to opposite disparities may be locally paired for some computational purpose. In the example of Figure $9 H$, MU tuning was relatively flat while SU responses were broadly tuned to far disparities. This could happen if the disparity map contains singularities akin to the pinwheel centers of orientation maps (Bonhoeffer and Grinvald, 1991; DeAngelis and Newsome, 1999) and if these recordings were made near such a singularity.

MU and SU responses were both available for 67 recording sites in V3 and 153 sites in V3A. Figure 10 compares DDI values derived from MU and SU responses, with each data point representing measurements made at a particular recording site for a given surround disparity (three data points per recording site). Highly significant correlations were found between SU and MU DDIs in both V3 $(r=0.71, p<0.01)$ and V3A $(r=0.67, p<$ 0.01 ), suggesting that neurons with a similar degree of disparity selectivity are clustered in these areas. Whereas this was expected from previous work in V3 (Adams and Zeki, 2001), it is a novel 
- both DDI significant

$\triangle \mathrm{MU} D D I$ significant $\nabla$ SU DDI significant

A $\square$ neither DDI significant

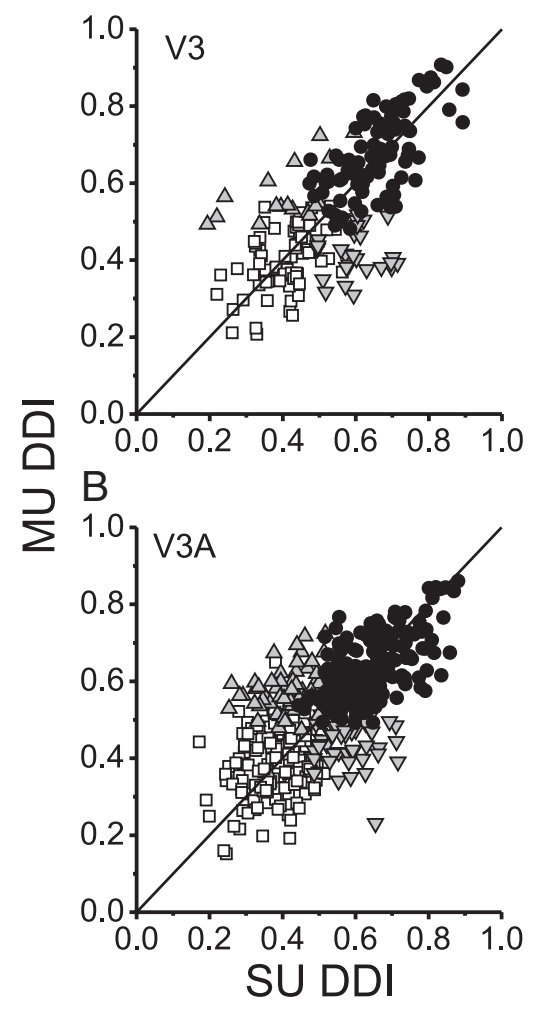

Figure 10. Comparison of DDl values for SU and MU responses from areas V3 $(\boldsymbol{A})$ and V3A $(\boldsymbol{B})$. Black circles, Both SU and MU tuning are significant; gray upward triangles, only MU disparity tuning is significant; gray downward triangles, only SU tuning is significant; white squares, neither SU nor MU tuning is significant. Solid lines indicate unity slope diagonals.

finding for $\mathrm{V} 3 \mathrm{~A}$. The correlations we found in $\mathrm{V} 3$ and $\mathrm{V} 3 \mathrm{~A}$ are similar to those found in V4 $[r=0.64$ (Tanabe et al., 2005), but $r=0.41$ (Watanabe et al., 2002) $]$ and MT $[r=0.66$ (DeAngelis and Newsome, 1999)] but higher than that found in V1 $[r=0.37$ (Prince et al., 2002b)].

To further characterize the similarity between SU and MU disparity-tuning, we selected pairs of SU and MU tuning curves that are both significant (Fig. 10, 85 and 183 tuning pairs from V3 and V3A, respectively, filled circles) and fitted Gabor functions to the MU tuning curves in the same manner as we did for the SU data. Figure 11 compares tuning parameters extracted from $\mathrm{Ga}$ bor fits to SU and MU responses that were deemed to be good fits (see Materials and Methods). Disparity preferences were generally well matched between SU and MU tuning in both V3 (Fig. $11 A$ ) and V3A (Fig. $11 B$ ), although there are some notable exceptions such as the neuron of Figure $9 G$ (Fig. $11 A$, indicated by open symbols). Correlations between disparity preferences of SU and MU tuning were significant in both V3 $(r=0.53, p<0.01)$ and V3A $(r=0.86, p<0.01)$, indicating that neurons are clustered according to their disparity preference as would be expected if there are disparity columns in these areas. Both of these correlations are substantially higher than those found in V1 $[r=0.30$ (Prince et al., 2002b) ] and V4 [ $r=0.43$ (Watanabe et al., 2002); $r=0.30$ (Tanabe et al., 2005)] but lower than (in the case of V3) or similar to (in the case of V3A) that found in MT $[r=0.91$ (DeAngelis and Newsome, 1999)].
The phase parameters of the best-fitting Gabor functions were also moderately correlated between SU and MU tuning in V3 (circular-circular correlation, $r=0.23$ ) and in V3A (circularcircular correlation, $r=0.41$ ) (Fig. $11 C, D)$, although the correlation was significant only for V3A $(p<0.01)$ and not for V3 $(p=0.09)$. Similarly, disparity frequency parameters were generally clustered around the unity-slope diagonal, with a handful of notable outliers as shown in Figure 11, E and $F$. The outliers at low SU and high MU disparity frequencies (Fig. 11 E, F, e.g., open symbols) are due to relatively flat tuning that happened to be fitted well by a Gabor function that oscillated sinusoidally, such as those at surround disparities of $0^{\circ}$ and $0.5^{\circ}$ in Figure $9 H$. Without these few outliers (Fig. 11, open symbols), there were significant correlations in the disparity frequency parameter for both V3 $(r=0.31, p=0.015)$ and V3A $(r=0.59, p<0.01)$. Since the phase parameter determines the symmetry of the disparitytuning and the disparity frequency is inversely related to the width of tuning, these observations suggest that the shape of tuning is generally similar between SU and MU responses, as typified by the examples in Figure 9.

Finally, we examined whether shift ratios (derived from the peak/trough-shift model) for MU tuning are correlated with those for SU tuning (Fig. 11G,H). Although shift ratios for individual neurons are mostly distributed around 0 (Fig. $7 A, B$ ), indicating that they encode absolute disparity, it is possible that the aggregate activity of neurons could signal relative disparity more strongly. However, we found that MU shift ratios are also mostly scattered around 0 and there is no significant difference in median shift ratios between the SU and MU distributions (ranksum test, $p=0.44$ for V3 and $p=0.50$ for V3A). There was a moderate correlation between $\mathrm{SU}$ and MU shift ratios in $\mathrm{V} 3$ $[r=0.55, p<0.01$ (Fig. $11 G$ ) $]$ and no significant correlation in V3A $[r=0.10, p=0.37$ (Fig. $11 \mathrm{H})]$. These findings suggest that the extraction of relative disparity signals from V3 and V3A neurons would require computations that are substantially more complex than just pooling the responses of nearby neurons tuned to binocular disparity.

\section{Discussion}

In this study, we examined basic disparity-tuning properties of neurons in V3 and V3A and compared them with similar data from neurons in other areas. Our results show that disparitytuning in $\mathrm{V} 3$ and $\mathrm{V} 3 \mathrm{~A}$ is not fundamentally different from that in other early and mid-level visual areas. We also measured the relative disparity-tuning of these neurons using the same measurement and analysis methods used in previous studies of other visual areas. We found that most neurons in V3 and V3A encode absolute, not relative, disparity. This finding contrasts with the results of fMRI studies that seem to show that V3 and V3A are activated by stimuli that are rich in relative disparity information (Tsao et al., 2003) (see also Backus et al., 2001). Finally, we found that neurons with similar disparity-tuning properties are clustered in V3 and V3A, which is a novel finding for V3A and confirms the results from a previous study of V3 neurons in anesthetized macaques (Adams and Zeki, 2001).

\section{Disparity-tuning properties of neurons in V3 and V3A}

Previously, only a few studies have examined disparity processing in V3 or V3A. Poggio et al. (1988) measured disparity-tuning of neurons in $\mathrm{V} 3 / \mathrm{V} 3 \mathrm{~A}$ of alert fixating monkeys using solid figure stereograms. They report that $\sim 80 \%$ of neurons in V3/V3A were selective for binocular disparity, which is substantially greater than what we have found ( $56 \%$ for V3, $53 \%$ for V3A). Note, 


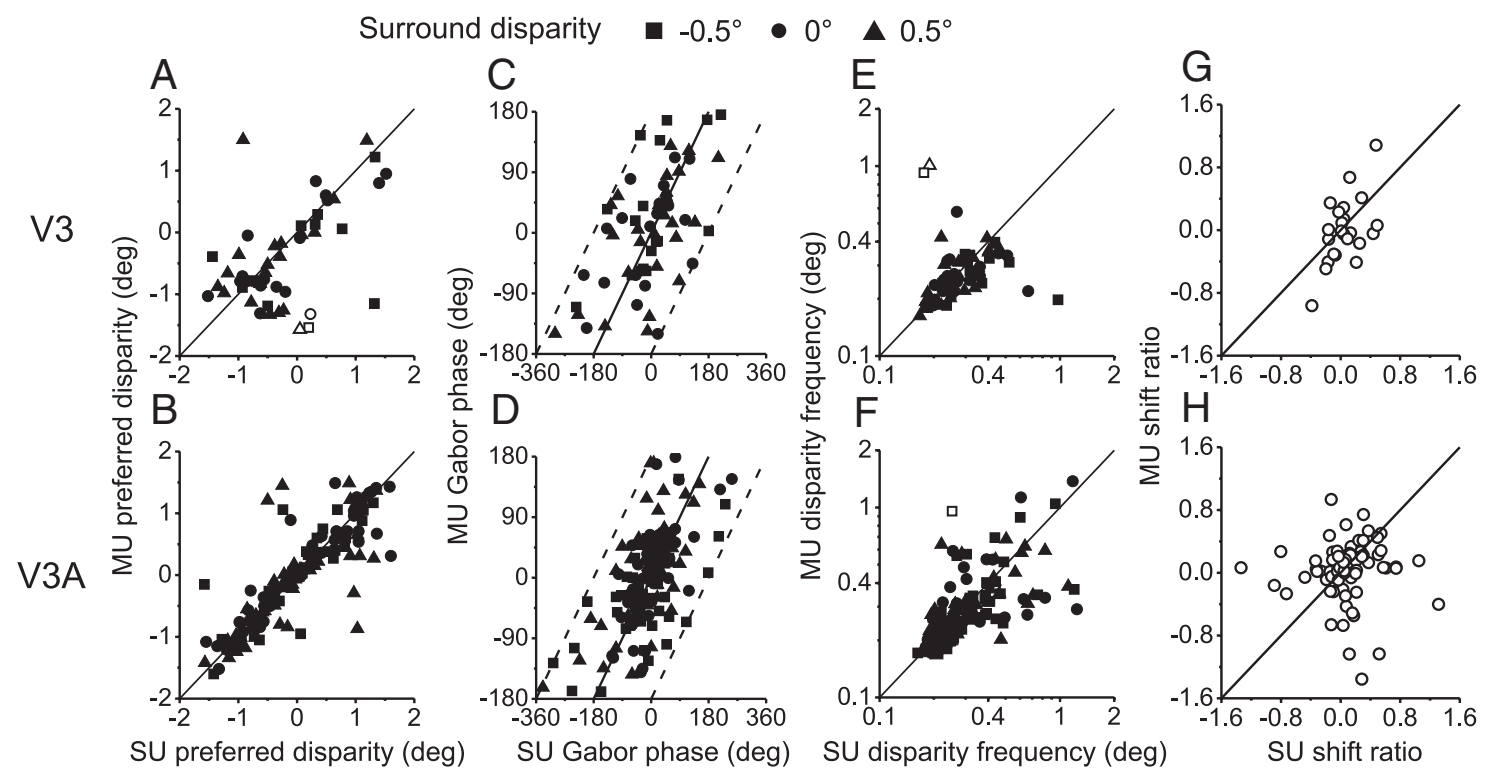

Figure 11. Comparisons of MU and SU disparity-tuning properties for surround disparities of $-0.5^{\circ}$ (squares), $0^{\circ}$ (circles), and $0.5^{\circ}$ (triangles) in $V 3(\boldsymbol{A}, \boldsymbol{C}, \boldsymbol{E}$, and $\boldsymbol{G})$ and $\mathrm{V} 3 \mathrm{~A}(\boldsymbol{B}, \boldsymbol{D}, \boldsymbol{F}$, and $\boldsymbol{H})$. Solid lines indicate unity slope diagonals. Open symbols in $\boldsymbol{A}, \boldsymbol{E}$, and $\boldsymbol{F}$ are outliers described in the text. $\boldsymbol{A}, \boldsymbol{B}$, Preferred disparity (V3: $N=51 ; \mathrm{V} 3 \mathrm{~A}: N=150)$. Preferred disparities beyond the stimulus disparity range (13 and 11 data points for V3 and V3A, respectively) were excluded (see Materials and Methods). $C$, $\mathbf{D}$, Gabor phase (V3: $N=64 ; \mathrm{V} 3 \mathrm{~A}: N=161)$. Since phase is a circular variable, it is bounded by the dashed lines. $\boldsymbol{E}, \boldsymbol{F}$, Disparity frequency $(\mathrm{V} 3: N=64 ; \mathrm{V3A}: N=161) . \boldsymbol{G}, \boldsymbol{H}$, Shift ratio based on the peak/trough-shift model $(\mathrm{V} 3: N=23 ; \mathrm{V} 3 \mathrm{~A}: N=78)$. In $\boldsymbol{H}$, three outliers beyond the range of the axes are not shown. deg, Degrees $\left({ }^{\circ}\right)$.

however, that this discrepancy could be due to different criteria for establishing that neurons are disparity selective, since Poggio et al. (1988) did not state their criterion. There was also no quantitative summary of disparity-tuning properties in that study, and data from V3 and V3A were grouped together, which makes comparisons between their data and ours difficult. Two other previous studies have examined disparity-tuning of V3 neurons in anesthetized monkeys (Felleman and Van Essen, 1987; Adams and Zeki, 2001). Both of these studies found examples of disparity-tuning similar to those described by Poggio et al. (1988), and the proportion of disparity-selective neurons was comparable to what we found in V3. Like Poggio et al. (1988), quantitative summaries were not provided in these studies, and further comparisons with our data are not possible. Thus, our study provides the first rigorous quantitative examination of disparity selectivity in areas V3 and V3A.

Our quantitative analysis also enabled direct comparisons with data from other visual areas. If $\mathrm{V} 3$ or $\mathrm{V} 3 \mathrm{~A}$ plays special roles in stereoscopic depth processing, we might expect to find disparity-tuning properties in these areas that are distinct, like the distribution of DDI values in MT (Fig. 2C) or the distribution of disparity preferences in V1 (Fig. 3C). However, we found that population distributions of DDI, preferred disparity, Gabor phase, and disparity frequency for V3 and V3A are similar to those observed in other early or mid-level visual areas. Therefore, at least with respect to the parameters we examined, V3 and V3A do not seem to show disparity-tuning that is radically different from other visual areas.

Finally, we found that $\mathrm{SU}$ and MU disparity-tuning at most recording sites was similar. This suggests that neurons in V3 and V3A with similar disparity-tuning properties are clustered. Poggio et al. (1988) noted that neurons with disparity selectivity tended to occur in clusters in V3/V3A. However, they did not directly examine whether nearby neurons generally shared similar disparity preferences. Adams and Zeki (2001) specifically addressed whether or not V3 is organized according to disparity selectivity. They found that neurons with similar disparity preferences are clustered and are organized into vertical columns. Our finding confirms their results in V3 and provides the first evidence of a topographic organization for disparity in V3A.

\section{Coding of absolute and relative disparity}

In search of cortical loci that compute relative disparity, previous studies from multiple laboratories have measured the tuning of single neurons for relative disparity in visual areas V1, V2, V4, and MT (Cumming and Parker, 1999; Thomas et al., 2002; Uka and DeAngelis, 2006; Umeda et al., 2007). All of these studies used similar stimuli and analysis methods so that direct comparisons of the results are possible. Combined with our findings, these studies show that early visual areas (V1, V2, and V3) and areas in the dorsal stream (V3A and MT) mainly carry absolute disparity signals, whereas area V4 in the ventral stream codes both relative and absolute disparities (see also Neri, 2005; Parker, 2007). Because absolute disparity is useful for driving eye movements (Rashbass and Westheimer, 1961; Cumming and Judge, 1986) while relative disparity is useful for fine depth judgments (Westheimer, 1979; Prince et al., 2000), these results are consistent with the notion that the dorsal stream is involved in visually guided action whereas the ventral stream is involved in perceptual identification of objects (Goodale and Milner, 1992).

However, these results need to be interpreted with some caution (Anzai and DeAngelis, 2010). All of these studies used a specific stimulus configuration, namely center-surround stimuli. In other words, these studies implicitly assumed that the computation of relative disparity involves some form of centersurround receptive field organization. However, there may be other mechanisms that could encode relative disparity. For example, von der Heydt et al. (2000) showed that some neurons in V2 respond to stereoscopically defined edges within the classical receptive field, suggesting that they signal disparity differences within the receptive field without invoking surround mechanisms (see also Bredfeldt and Cumming, 2006). Similarly, some 
MT neurons show 3D surface orientation selectivity that is tolerant to variations in mean disparity and does not depend on surround inhibition (Nguyenkim and DeAngelis, 2003), suggesting that these neurons can signal disparity gradients within the receptive field. This might be considered a different form of relative disparity selectivity (but see Bridge and Cumming, 2008).

From a computational stand point, relative disparity may not be fully represented until stereo correspondence is established and surface representations are created. Unless the correspondence problem is solved and absolute disparities are estimated accurately, relative disparity may not be estimated correctly. There is evidence that the stereo correspondence problem is largely solved in V4 and the inferior temporal area (Janssen et al., 2003; Tanabe et al., 2004). This suggests that robust relative disparity signals may become available in these areas, which is consistent with the findings of Umeda et al. (2007) in V4.

\section{Are areas V3 and V3A specialized for stereoscopic depth processing?}

Functional MRI studies have reported that V3 and V3A are strongly activated by stimuli that are rich in relative disparities (Tsao et al., 2003) (see also Backus et al., 2001). Our findings appear to be at odds with these studies, as we did not find any clear evidence that neurons in V3 and V3A are specialized to encode relative disparity. This was the case for both SU and MU activity, the latter of which may be more closely related to fMRI signals (Logothetis et al., 2001). We also did not find any basic disparity-tuning characteristics in V3 and V3A that are sufficiently distinct from those of other visual areas to indicate that these areas are specialized for stereoscopic depth processing.

It is not clear how to account for the discrepancy between our findings and some previous fMRI results. One possibility is that the stimuli used in fMRI studies were not effective in isolating responses due to relative disparity. Tsao et al. (2003) used disparity checkerboard stimuli, which contained both relative and absolute disparities, and found strong activations in both V3 and V3A of macaques. In contrast, Neri et al. (2004) examined sensitivities to absolute and relative disparities of various cortical areas in humans by measuring effects of adaptation to each type of disparity. They found that V3A exhibited more adaption to absolute disparity than relative disparity, suggesting that neurons in this area are mainly sensitive to absolute disparity, which agrees with our finding in monkeys.

It is also possible that V3A is involved in surface segmentation processes. Since disparity is a robust cue for segmentation, the V3A activity observed in Tsao et al. (2003) may be associated with segmentation rather than relative depth per se. To examine this possibility, Tsao et al. (2003) measured fMRI signals for orientation-defined checkerboard stimuli. They found that V3A was not activated and concluded that $\mathrm{V} 3 \mathrm{~A}$ is not concerned with surface segmentation in general but rather with 3D scene layout. In contrast, other fMRI studies have reported that V3A is activated by stimuli that contain nonstereo segmentation cues such as orientation-defined checkerboards (Kastner et al., 2000) and illusory contour-defined shapes (Mendola et al., 1999). In particular, the latter study found a significant overlap between regions activated by stereo-defined and illusory contour-defined shapes in V3A, suggesting that V3A may be involved in surface segmentation. It remains to be seen if single neurons in $\mathrm{V} 3 \mathrm{~A}$ process both stereo and nonstereo cues in a manner consistent with surface segmentation.

\section{References}

Adams DL, Zeki S (2001) Functional organization of macaque V3 for stereoscopic depth. J Neurophysiol 86:2195-2203.

Anzai A, DeAngelis GC (2010) Neural computations underlying depth perception. Curr Opin Neurobiol 20:367-375.

Backus BT, Fleet DJ, Parker AJ, Heeger DJ (2001) Human cortical activity correlates with stereoscopic depth perception. J Neurophysiol 86: 2054-2068.

Bonhoeffer T, Grinvald A (1991) Iso-orientation domains in cat visual cortex are arranged in pinwheel-like patterns. Nature 353:429-431.

Bredfeldt CE, Cumming BG (2006) A simple account of cyclopean edge responses in macaque v2. J Neurosci 26:7581-7596.

Bridge H, Cumming BG (2008) Representation of binocular surfaces by cortical neurons. Curr Opin Neurobiol 18:425-430.

Chen A, Gu Y, Takahashi K, Angelaki DE, Deangelis GC (2008) Clustering of self-motion selectivity and visual response properties in macaque area MSTd. J Neurophysiol 100:2669-2683.

Cumming BG, DeAngelis GC (2001) The physiology of stereopsis. Annu Rev Neurosci 24:203-238.

Cumming BG, Judge SJ (1986) Disparity-induced and blur-induced convergence eye movement and accommodation in the monkey. J Neurophysiol 55:896-914.

Cumming BG, Parker AJ (1999) Binocular neurons in V1 of awake monkeys are selective for absolute, not relative, disparity. J Neurosci 19:5602-5618.

DeAngelis GC, Newsome WT (1999) Organization of disparity-selective neurons in macaque area MT. J Neurosci 19:1398-1415.

DeAngelis GC, Uka T (2003) Coding of horizontal disparity and velocity by MT neurons in the alert macaque. J Neurophysiol 89:1094-1111.

Durand JB, Zhu S, Celebrini S, Trotter Y (2002) Neurons in parafoveal areas V1 and V2 encode vertical and horizontal disparities. J Neurophysiol 88:2874-2879.

Efron B, Tibshirani R (1993) An introduction to the bootstrap. New York: Chapman and Hall.

Erkelens CJ, Collewijn H (1985) Motion perception during dichoptic viewing of moving random-dot stereograms. Vision Res 25:583-588.

Felleman DJ, Van Essen DC (1987) Receptive field properties of neurons in area V3 of macaque monkey extrastriate cortex. J Neurophysiol 57:889-920.

Fisher NI, Marron JS (2001) Mode testing via the excess mass estimate. Biometrika 88:499-517.

Gattass R, Sousa AP, Gross CG (1988) Visuotopic organization and extent of V3 and V4 of the macaque. J Neurosci 8:1831-1845.

Gonzalez F, Perez R (1998) Neural mechanisms underlying stereoscopic vision. Prog Neurobiol 55:191-224.

Goodale MA, Milner AD (1992) Separate visual pathways for perception and action. Trends Neurosci 15:20-25.

Hubel DH, Wiesel TN (1962) Receptive fields, binocular interaction and functional architecture in the cat's visual cortex. J Physiol 160:106-154.

Hubel DH, Wiesel TN (1963) Shape and arrangement of columns in cat's striate cortex. J Physiol 165:559-568.

Janssen P, Vogels R, Liu Y, Orban GA (2003) At least at the level of inferior temporal cortex, the stereo correspondence problem is solved. Neuron 37:693-701

Julesz B (1971) Foundation of cyclopean perception. Chicago: University of Chicago.

Kastner S, De Weerd P, Ungerleider LG (2000) Texture segregation in the human visual cortex: A functional MRI study. J Neurophysiol 83:2453-2457.

Logothetis NK, Pauls J, Augath M, Trinath T, Oeltermann A (2001) Neurophysiological investigation of the basis of the fMRI signal. Nature 412:150-157.

Mendola JD, Dale AM, Fischl B, Liu AK, Tootell RB (1999) The representation of illusory and real contours in human cortical visual areas revealed by functional magnetic resonance imaging. J Neurosci 19:8560-8572.

Mountcastle VB (1997) The columnar organization of the neocortex. Brain 120:701-722.

Movshon JA, Thompson ID, Tolhurst DJ (1978) Spatial and temporal contrast sensitivity of neurones in areas 17 and 18 of the cat's visual cortex. J Physiol 283:101-120.

Neri P (2005) A stereoscopic look at visual cortex. J Neurophysiol 93:1823-1826. 
Neri P, Bridge H, Heeger DJ (2004) Stereoscopic processing of absolute and relative disparity in human visual cortex. J Neurophysiol 92:1880-1891.

Nguyenkim JD, DeAngelis GC (2003) Disparity-based coding of threedimensional surface orientation by macaque middle temporal neurons. J Neurosci 23:7117-7128.

Parker AJ (2007) Binocular depth perception and the cerebral cortex. Nat Rev Neurosci 8:379-391.

Poggio GF, Gonzalez F, Krause F (1988) Stereoscopic mechanisms in monkey visual cortex: binocular correlation and disparity selectivity. J Neurosci 8:4531-4550.

Prince SJ, Pointon AD, Cumming BG, Parker AJ (2000) The precision of single neuron responses in cortical area $\mathrm{V} 1$ during stereoscopic depth judgments. J Neurosci 20:3387-3400.

Prince SJ, Cumming BG, Parker AJ (2002a) Range and mechanism of encoding of horizontal disparity in macaque V1. J Neurophysiol 87: 209-221.

Prince SJ, Pointon AD, Cumming BG, Parker AJ (2002b) Quantitative analysis of the responses of $\mathrm{V} 1$ neurons to horizontal disparity in dynamic random-dot stereograms. J Neurophysiol 87:191-208.

Rashbass C, Westheimer G (1961) Disjunctive eye movements. J Physiol 159:339-360.

Schiller PH, Finlay BL, Volman SF (1976) Quantitative studies of single-cell properties in monkey striate cortex. III. Spatial frequency. J Neurophysiol 39:1334-1351.

Smith AT, Singh KD, Williams AL, Greenlee MW (2001) Estimating receptive field size from fMRI data in human striate and extrastriate visual cortex. Cereb Cortex 11:1182-1190.

Tanabe S, Umeda K, Fujita I (2004) Rejection of false matches for binocular correspondence in macaque visual cortical area V4. J Neurosci 24: $8170-8180$

Tanabe S, Doi T, Umeda K, Fujita I (2005) Disparity-tuning characteristics of neuronal responses to dynamic random-dot stereograms in macaque visual area V4. J Neurophysiol 94:2683-2699.

Thomas OM, Cumming BG, Parker AJ (2002) A specialization for relative disparity in V2. Nat Neurosci 5:472-478.

Tsao DY, Vanduffel W, Sasaki Y, Fize D, Knutsen TA, Mandeville JB, Wald LL, Dale AM, Rosen BR, Van Essen DC, Livingstone MS, Orban GA, Tootell RB (2003) Stereopsis activates V3A and caudal intraparietal areas in macaques and humans. Neuron 39:555-568.

Uka T, DeAngelis GC (2003) Contribution of middle temporal area to coarse depth discrimination: comparison of neuronal and psychophysical sensitivity. J Neurosci 23:3515-3530.

Uka T, DeAngelis GC (2006) Linking neural representation to function in stereoscopic depth perception: roles of the middle temporal area in coarse versus fine disparity discrimination. J Neurosci 26:6791-6802.

Umeda K, Tanabe S, Fujita I (2007) Representation of stereoscopic depth based on relative disparity in macaque area V4. J Neurophysiol 98: 241-252.

Van Essen DC, Zeki SM (1978) The topographic organization of rhesus monkey prestriate cortex. J Physiol 277:193-226.

Van Essen DC, Drury HA, Dickson J, Harwell J, Hanlon D, Anderson CH (2001) An integrated software suite for surface-based analyses of cerebral cortex. J Am Med Inform Assoc 8:443-459.

von der Heydt R, Zhou H, Friedman HS (2000) Representation of stereoscopic edges in monkey visual cortex. Vision Res 40:1955-1967.

Watanabe M, Tanaka H, Uka T, Fujita I (2002) Disparity-selective neurons in area V4 of macaque monkeys. J Neurophysiol 87:1960-1973.

Westheimer G (1979) Cooperative neural processes involved in stereoscopic acuity. Exp Brain Res 36:585-597.

Wheatstone C (1838) On some remarkable, and hitherto unobserved, phenomena of binocular vision. Philos Trans R Soc Lond 128:371-394.

Zeki SM (1978) The third visual complex of rhesus monkey prestriate cortex. J Physiol 277:245-272. 